\title{
FACTORS AFFECTING LIQUID CHROMATOGRAPHY TANDEM-MASS SPECTROMETRY ANALYSIS OF 6-MERCAPTOPURINE AND 6-THIOGUANINE IN DRIED BLOOD SPOTS
}

\author{
YAHDIANA HARAHAP*, DIMAS AGUS PUTERA HARDIJANTO, DELLY RAMADON
}

Faculty of Pharmacy, Universitas Indonesia, Depok 16424, Indonesia. Email: yahdiana03@yahoo.com

Received: 08 June 2018, Revised and Accepted: 09 August 2018

ABSTRACT

Objective: This study aimed to determine the effects of the method of internal standard addition, spotting volume, paper type, and sample storage temperature on 6-mercaptopurine, and 6-thioguanine on liquid chromatography tandem-mass spectrometry (LC-MS/MS) bioanalysis methods using dried blood spot (DBS).

Methods: Blood samples were spotted on CAMAG DBS paper and a Perkin Elmer 226 sample collection device (paper) and extracted into methanol containing 5-fluorouracil as an internal standard. The separation was performed on a water acquity ultra high-performance LC BEH Amide $1.7 \mu \mathrm{m}$ $(2.1 \mathrm{~mm} \times 100 \mathrm{~mm})$ column with a mobile phase of $0.2 \%$ formic acid in water $-0.1 \%$ formic acid in acetonitrile methanol with gradient elution at a flow rate of $0.2 \mathrm{~mL} / \mathrm{min}$.

Results: The step at which the internal standard was added (blood, spot on DBS card, or extraction solution) affected the chromatogram. Differences in paper types and blood volumes significantly affected $(\mathrm{p}<0.05)$ the percent coefficient of variation, whereas differences in blood hematocrit significantly affected the peak area ratio.

Conclusion: The method of internal standard addition affected the chromatograms in this study. The best chromatogram was observed when the Internal Standard was added to the extracting solution. The card type also affected the analysis, so it is recommended to use the same card during sample analysis.

Keywords: 5-fluorouracil, 6-mercaptopurine, 6-thioguanine, Dried blood spot, Hematocrit, Liquid chromatography tandem-mass spectrometry.

(C) 2018 The Authors. Published by Innovare Academic Sciences Pvt Ltd. This is an open access article under the CC BY license (http://creativecommons. org/licenses/by/4. 0/) DOI: http://dx.doi.org/10.22159/ijap.2018.v10s1.44

\section{INTRODUCTION}

The dried blood spot (DBS) method has been used as a sampling technique in therapeutic drug monitoring of some drugs in clinical practice. This sampling method offers several advantages over venipuncture, such as more convenient for the patient because it is less invasive than taking blood using a sterile lancet needle on the fingers, low volume, easy storage, storage stability, and reduced risk of infection [1,2]. However, several factors should be considered when a DBS method is developed and validated. These include the small blood volume, varying hematocrit (HT) values in patients undergoing chemotherapy, the presence of metabolites resulting from metabolism, and the different paper types to be used in the analysis. Since the HT represents the relative volume of the red blood cells in the blood, it also affects the viscosity of the blood. Therefore, the spread of the blood drop on the DBS card is influenced by the HT of the blood; high HT forms smaller spots and vice versa.

Different types of card matrices in combination with varying HT values may have different effects on blood spot spreading and extraction recovery of the substances. In several previous studies, there were differences in the performance of five paper types on the immunosuppressant analysis in the DBS sample [3]. Differences in HT values have affected the diameter of the spot but were not found to affect the analysis [4]. The blood volume also has not been found to affectanalysis results [5].Storagetemperatures have beenfound to affect sample stability after $>6$ months [6]. Study results are highly depend on the drugs to be analyzed.

The objective of this study was to determine the effects of the method of internal standard addition, spotting volume, paper type, and sample storage temperature on 6-mercaptopurine (6-MP), and 6-thioguanine (6-TG) on liquid chromatography tandem-mass spectrometry (LC-MS/ MS) bioanalysis methods using DBS.

\section{METHODS}

\section{Instrumentation}

An ultra-high-performance LC (UPLC) system (Waters Acquity UPLC $\mathrm{H}$-Class) coupled with a tandem mass spectrometer (Waters Xevo TQD Triple Quadrupole) equipped with electrospray ionization (ESI) source was used. The chromatographic separations were performed on a waters acquity UPLC Class BEH amide column $(1.7 \mu \mathrm{m}$ particle size; $100 \mathrm{~mm}$ (L) $\times 2.1 \mathrm{~mm}$ [I.D.]) at $25^{\circ} \mathrm{C}$. The mobile phase conditions were as follows: Reservoir $\mathrm{A}=0.2 \%$ formic acid in water; reservoir $\mathrm{B}=0.1 \%$ formic acid in acetonitrile methanol; flow rate of $0.2 \mathrm{~mL} / \mathrm{min}$. The mass spectrometer parameters for scans in positive and negative ESI mode were optimized. The mass to charge $(\mathrm{m} / \mathrm{z})$ ratios of 6-MP, 6-TG, and 5-fluorouracil (5-FU) are $153.10>119.09 ; 168.09>107.06$; and $129.15>42.05$, respectively.

\section{Chemical and reagents}

6-MP, 6-TG, and 5-FU (IS) were purchased from Sigma-Aldrich. Acetonitrile (HPLC grade), methanol (HPLC grade), and formic acid were purchased from Merck. Ultra-pure water was collected from a Sartorius water filter. CAMAG DBS paper and Perkin Elmer 226 sample collection devices (paper) were used to hold the blood spots. Whole blood with HT percentages of $35 \%, 38 \%, 40 \%, 42 \%, 45 \%$, and $48 \%$ was procured from the Indonesian Red Cross.

\section{Preparation of stock solution}

A 5-mg sample from each of pure 6-MP, 6-TG, and 5-FU was weighed $( \pm 0.1 \mathrm{mg})$ and placed into separate $5.0-\mathrm{mL}$ flasks. The substances were then dissolved in $2 \mathrm{~mL}$ of $1 \mathrm{~N} \mathrm{NH}_{4} \mathrm{OH}$ to which $3 \mathrm{~mL}$ of methanol was 
added to obtain standard solutions of $1.0 \mathrm{mg} / \mathrm{mL}$. Subsequent working solutions were diluted in $\mathrm{NH}_{4} \mathrm{OH}-$ methanol, 40:60 v/v.

Preparation of calibration standards and quality control (QC) samples

A $900-\mu \mathrm{L}$ aliquot of blood was spiked with $100 \mu \mathrm{L}$ of each drug to prepare working solutions $(10 \mathrm{ppm})$ with calibrated concentrations of 6-MP and 6-TG of 25, 50, 100, 200, 500, 800, and $1000 \mathrm{ng} / \mathrm{mL}$ and QC concentrations of 100,500 , and $800 \mathrm{ng} / \mathrm{mL}$. The solutions were stored at $-20^{\circ} \mathrm{C}$ until analysis.

\section{Sample preparation}

An 8-mm diameter disk was punched out from the blood spots for extraction and placed into a 4-mL microcentrifuge tube. A 100- $\mu \mathrm{L}$ aliquot of 100-ppm IS in $4 \mathrm{~mL}$ of methanol was added to each tube. The samples were vortex mixed for $5 \mathrm{~min}$ followed by sonication for $20 \mathrm{~min}$ and centrifugation for $5 \mathrm{~min}$ at $500 \mathrm{~g}$. The samples were evaporated for $30 \mathrm{~min}$. The supernatants were then transferred to autosampler vials, and $10-\mu \mathrm{L}$ samples were injected into the UPLC.

\section{Effect of IS provisions}

The compound 5-FU was used as an IS because it has a similar structure and physicochemical properties as those of 6-MP and 6-TG but can be separated by chromatography. The addition of the IS was performed by adding $100 \mu \mathrm{L}$ of $5-\mathrm{FU}$ at $100 \mu \mathrm{g} / \mathrm{mL}$ to each of the following: The blood, DBS cards, and extraction solution. The differences in the IS chromatograms were assessed to determine at what step the IS should be added.

\section{Effect of HT, card types, and volume}

6-MP and 6-TG at concentrations of $25 \mathrm{ng} / \mathrm{mL}$ (lower limit of quantification) and $1000 \mathrm{ng} / \mathrm{mL}$ (upper limit of quantification) in blood aliquots with HT levels of $35 \%, 38 \%, 40 \%, 42 \%, 45 \%$, and $48 \%$ were spotted on CAMAG DBS paper and Perkin Elmer 226 sample collection devices at volumes 10, 20, 40, and $60 \mu \mathrm{L}$.

\section{Effect of storage temperature}

Samples containing 6-MP and 6-TG at a concentration of $100 \mu \mathrm{g} / \mathrm{mL}$ (QC low) and $800 \mathrm{ng} / \mathrm{mL}$ (QC high) were spotted onto the cards and stored at room temperature $\left(25^{\circ} \mathrm{C}\right)$ and $-20^{\circ} \mathrm{C}$ for 16 days. The samples were then extracted and analyzed. The stability of the substances was observed by calculating the percentage difference.

\section{RESULTS AND DISCUSSION}

\section{Effect of IS provisions}

In general, an IS is added to an extraction solution. However, there are several ways to add an IS, such as in the blood or spotted on a DBS card [7-9]. The chromatograms of the IS, when added to the blood or spotted on the DBS paper, were poor, which indicated that the IS should be added to the extraction solution. The results suggested that some of the IS were permanently absorbed into the cards, which decreased the amount of IS that was extracted, injected into the UPLC, and detected by MS/MS.

\section{Effect of card types}

Each type of DBS card is made from different raw materials, which can affect performance during analysis. Significant correlations $(p<0.05)$ between paper types and the mean measured concentrations were seen in the analysis of 6-MP $25 \mathrm{ng} / \mathrm{mL}$ and 6-TG $1000 \mathrm{ng} / \mathrm{mL}$, whereas the result showed no correlation (Table 1). The bivariate analysis showed that the mean measured concentrations were greater on the Perkin Elmer 226 paper. The difference in measured concentrations between the Perkin Elmer 226 and DBS CAMAG papers was caused by the differences in the raw materials used to make the paper. The compounds were more strongly absorbed into the Perkin Elmer 226 paper than into the DBS CAMAG paper, so compounds were more easily partitioned into the solvent during the extraction process. However, the correlation between DBS card types and coefficients of variation could not be determined because the number of replicates was too small to determine the standard deviation and coefficient of variation. Therefore, conclusions regarding the best type of DBS paper for sample analysis could not be drawn [10-12].

\section{Effects of HT and volume on blood spread}

HT will affect blood spread in the cards, so it will affect how many analytes in blood are analyzed. In Figs. 1, 2 and Table 2, the areas of

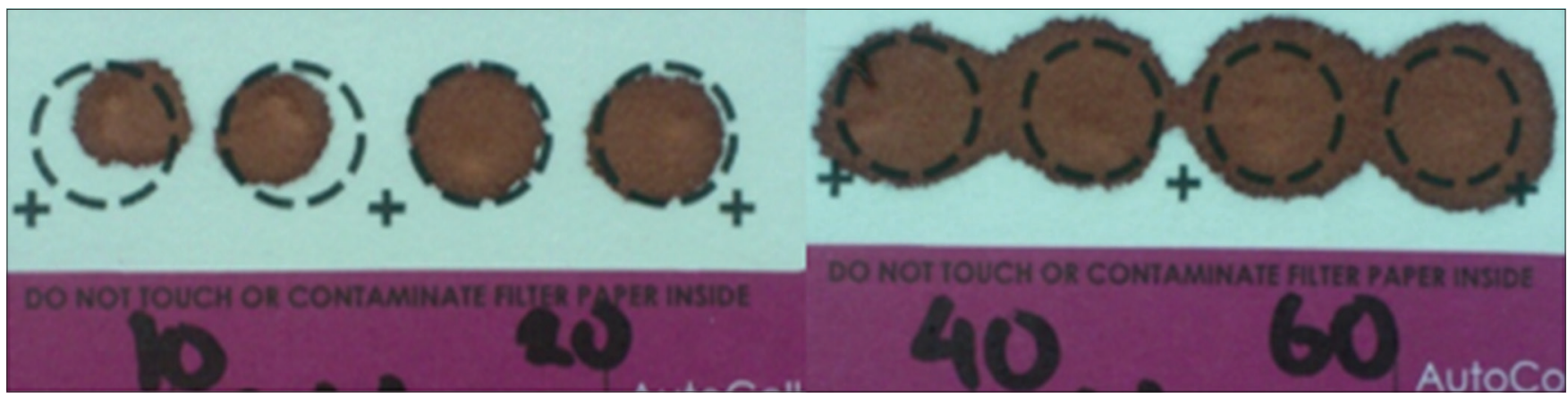

Fig. 1: Blood spots with hematocrit of $35 \%$

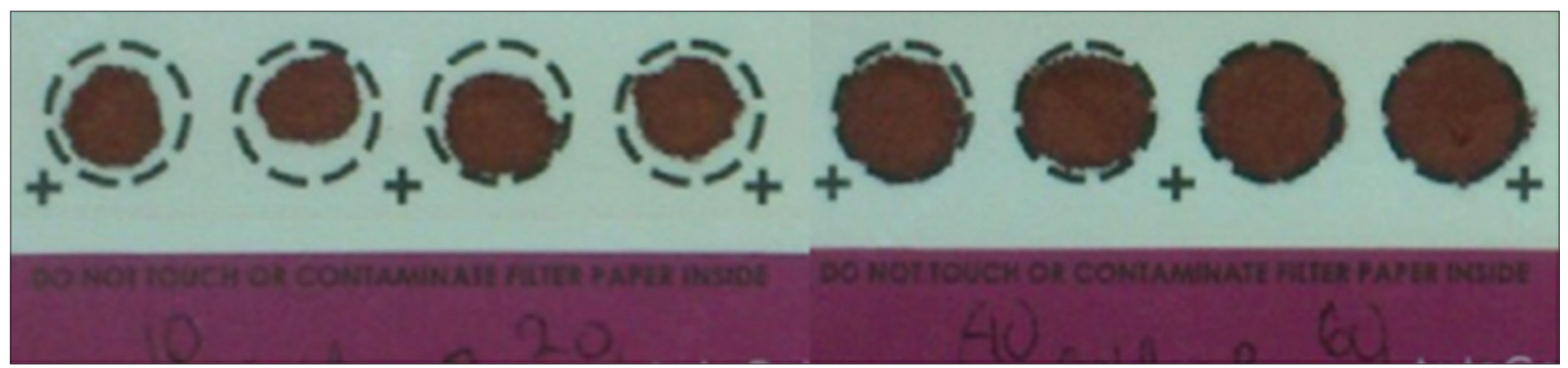

Fig. 2: Blood spots with hematocrit of $48 \%$ 
the spots were affected by HT values. Figs. 3 and 4 show that the HT and blood volume affected the peak area ratio (PAR) and coefficient of variation.

For HT levels of $35 \%, 38 \%, 40 \%$, and $42 \%$, PAR increased up to a blood volume of $40 \mu \mathrm{L}$ but decreased at $60 \mu \mathrm{L}$. This phenomenon occurred because the blood spot diameters were too large, so not all analytes were included within the punched-out paper area. For HT levels of $45 \%$ and $48 \%$, PAR continued to increase up to $60 \mu \mathrm{L}$ because the blood

Table 1: Bivariate analysis of DBS cards

\begin{tabular}{llll}
\hline Cards & Analysis & Mean & Difference \\
\hline 6-MP 25 ng/mL & Pearson correlation & $-0.261^{*}$ & -0.193 \\
& Significant (2-tailed p value) & 0.027 & 0.105 \\
& $\mathrm{n}$ & 72 & 72 \\
6-TG $1000 \mathrm{ng} / \mathrm{mL}$ & Pearson correlation & $-0.234^{*}$ & -0.198 \\
& Significant (2-tailed p value) & 0.040 & 0.096 \\
& $\mathrm{n}$ & 72 & 72 \\
\hline
\end{tabular}

*Correlated. 6-MP: 6-mercaptopurine, 6-TG: 6-thioguanine, DBS: Dried blood spot

Table 2: Spot diameter data

\begin{tabular}{lllllll}
\hline Volume $(\boldsymbol{\mu l})$ & $\mathbf{3 5 \%}$ & $\mathbf{3 8 \%}$ & $\mathbf{H T}(\mathbf{m m}) \mathbf{4 0 \%}$ & $\mathbf{4 2 \%}$ & $\mathbf{4 5 \%}$ & $\mathbf{4 8 \%}$ \\
\hline 10 & 7 & 7 & 6 & 6 & 6 & 6 \\
20 & 8 & 8 & 8 & 7 & 7 & 7 \\
40 & 12 & 11 & 10 & 10 & 9 & 8 \\
60 & 14 & 13 & 12 & 11 & 10 & 9 \\
\hline
\end{tabular}

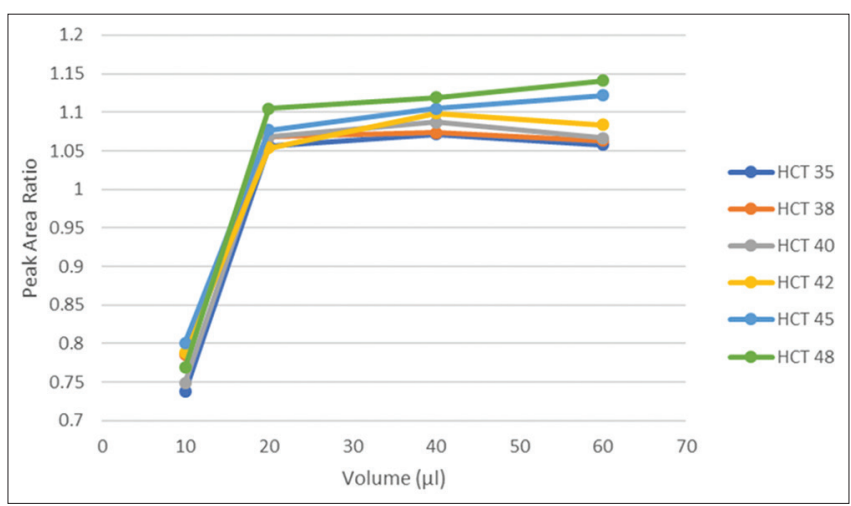

Fig. 3: Effect of volume and hematocrit on peak area ratio of 6-mercaptopurine applied at $25 \mathrm{ng} / \mathrm{mL}$ on a Perkin Elmer 226 sample collection device (paper)

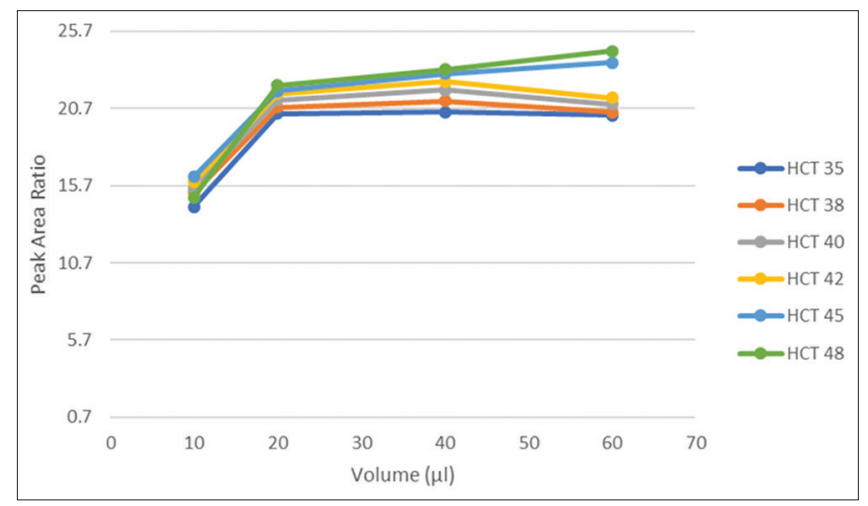

Fig. 4: Effect of volume and hematocrit on peak area ratio of 6-thioguanine applied at $1000 \mathrm{ng} / \mathrm{mL}$ on CAMAG dried blood spotpaper spread was not too large, which meant that all analytes were included in the punched-out paper area. At $10-\mu \mathrm{L}$ volumes, the spot diameters were too small, so the analytes present in the paper cards were insufficient for analysis.

\section{Effect of storage temperature}

DBS samples can be stored at room temperature. However, in some studies, storing the samples at lower temperatures has improved longterm stability. The percentage difference in the 6-MP and 6-TG samples stored at room temperature or at $-20^{\circ} \mathrm{C}$ varied by $\pm 15 \%$ for 16 days. This result showed that the samples were stable at these two temperatures and could be analyzed within 16 days. There were no significant differences in the amounts of compounds stored at the two temperatures for 16 days. Potential differences may be seen after longer storage times.

\section{CONCLUSION}

The best chromatogram was observed when the IS was added to the extracting solution. The card type also affected the analysis. Therefore, it is recommended to use the same card from the start of optimization to sample analysis. Re-validation must be performed when the card type is changed. The HT percentage affected the blood distribution and spread and correlated with the spotting volume. To obtain a constant result, the same spotting volume is required. DBS samples were analyzed after being stored for up to 16 days at room temperature and at $-20^{\circ} \mathrm{C}$ with similar results. However, the optimal storage temperature requires further investigation.

\section{CONFLICTS OF INTEREST}

All authors have none to declare

\section{REFERENCES}

1. Déglon J, Thomas A, Mangin P, Staub C. Direct analysis of dried blood spots coupled with mass spectrometry: Concepts and biomedical applications. Anal Bioanal Chem 2012;402:2485-98.

2. Evans C, Arnold M, Bryan P, Duggan J, James CA, Li W, et al. Implementing dried blood spot sampling for clinical pharmacokinetic determinations: Considerations from the IQ consortium microsampling working group. AAPS J 2015;17:292-300.

3. Koster RA, Botma R, Greijdanus B, Uges DR, Kosterink JG, Touw DJ, et al. The performance of five different dried blood spot cards for the analysis of six immunosuppressants. Bioanalysis 2015;7:1225-35.

4. Denniff P, Spooner N. The effect of hematocrit on assay bias when using DBS samples for the quantitative bioanalysis of drugs. Bioanalysis 2010;2:1385-95.

5. Liang X, Li Y, Barfield M, Ji QC. Study of dried blood spots technique for the determination of dextromethorphan and its metabolite dextrorphan in human whole blood by LC-MS/MS. J Chromatogr B Analyt Technol Biomed Life Sci 2009;877:799-806

6. Prentice P, Turner C, Wong MC, Dalton RN. Stability of metabolites in dried blood spots stored at different temperatures over a 2-year period. Bioanalysis 2013;5:1507-14.

7. World Health Organization. Flame Retardants: Tris (2-Butoxyethyl) Phosphate, Tris(2-Ethylhexyl) Phosphate and Tetrakis (Hydroxymethyl) Phosphonium Salt, Environmental Health Criteria 218. Geneva: World Health Organization; 2000. p. 1-130.

8. Weisz A, Milstein SR, Scher AL, Hepp NM. Colouring agents in cosmetics: Regulatory aspects and analytical methods. In: Analysis of Cosmetic Products. Ch. 7. $2^{\text {nd }}$ ed. Amsterdam: Elsevier; 2018. p. 123-57.

9. Ruiz NM, Perez MT, Diaz HM, Izquierdo M, Blanco M, Machado LY, et al. Determination of HIV-1 viral load on dried blood spot specimens. Biotechnol Apl 2014;31:146-9.

10. Lafreniere NM, Shih SC, Abu-rabie P, Jebrail MJ, Spooner N, Wheeler AR. Multiplexed extraction and quantitative analysis of pharmaceuticals from DBS samples using digital microfluidics. Bioanalysis 2014;6:307-18.

11. Spooner N, Ramakrishnan Y, Barfield M, Dewit O, Miller S. Use of DBS sample collection to determine circulating drug concentrations in clinical trials: Practicalities and considerations. Bioanalysis 2010;2:1515-22.

12. Abu-Rabie P. Direct analysis of DBS: Emerging and desirable technologies. Bioanalysis 2011;3:1675-8. 